\title{
Complete genome sequence of Jonesia denitrificans type strain (Prevot 55134 ${ }^{\mathrm{T}}$ )
}

\author{
Rüdiger Pukall ${ }^{1}$, Gabriele Gehrich-Schröter ${ }^{1}$, Alla Lapidus ${ }^{2}$, Matt Nolan², Tijana Glavina \\ Del Rio ${ }^{2}$, Susan Lucas², Feng Chen², Hope Tice², Sam Pitluck², Jan-Fang Cheng², Alex Co- \\ peland $^{2}$, Elizabeth Saunders ${ }^{2,3}$, Thomas Brettin ${ }^{2,3}$, John C. Detter ${ }^{2,3}$, David Bruce ${ }^{2,3}$, Lynne \\ Goodwin $^{2,3}$, Amrita Pati ${ }^{2}$, Natalia Ivanova ${ }^{2}$, Konstantinos Mavrommatis ${ }^{2}$, Galina Ovchinni- \\ kova $^{2}$, Amy Chen ${ }^{4}$, Krishna Palaniappan ${ }^{4}$, Miriam Land ${ }^{2,5}$, Loren Hauser ${ }^{2,5}$, Yun-Juan \\ Chang $^{2,5}$, Cynthia D. Jeffries ${ }^{2,5}$, Patrick Chain ${ }^{2,6}$, Markus Göker ${ }^{1}$, Jim Bristow ${ }^{2}$, Jonathan A. \\ Eisen $^{2,7}$, Victor Markowitz ${ }^{4}$, Philip Hugenholtz ${ }^{2}$, Nikos C. Kyrpides ${ }^{2}$, Hans-Peter Klenk ${ }^{{ }^{*}}$, and \\ Cliff $\mathrm{Han}^{2,3}$ \\ ${ }^{1}$ DSMZ - German Collection of Microorganisms and Cell Cultures GmbH, Braunschweig, \\ Germany \\ ${ }^{2}$ DOE Joint Genome Institute, Walnut Creek, California, USA \\ ${ }^{3}$ Los Alamos National Laboratory, Bioscience Division, Los Alamos, New Mexico, USA \\ ${ }^{4}$ Biological Data Management and Technology Center, Lawrence Berkeley National Labora- \\ tory, Berkeley, California, USA \\ ${ }^{5}$ Oak Ridge National Laboratory, Oak Ridge, Tennessee, USA \\ ${ }^{6}$ Lawrence Livermore National Laboratory, Livermore, California, USA \\ ${ }^{7}$ University of California Davis Genome Center, Davis, California, USA
}

*Corresponding author: Hans-Peter Klenk

Keywords: Actinobacteria, Actinomycetales, Micrococcineae, Jonesiaceae; Gram-positive, irregular, nonsporulating rods, ox blood

Jonesia denitrificans (Prevot 1961) Rocourt et al. 1987 is the type species of the genus Jonesia, and is of phylogenetic interest because of its isolated location in the actinobacterial suborder Micrococcineae. J. denitrificans is characterized by a typical coryneform morphology and is able to form irregular nonsporulating rods showing branched and club-like forms. Coccoid cells occur in older cultures. J. denitrificans is classified as a pathogenic organism for animals (vertebrates). The type strain whose genome is described here was originally isolated from cooked ox blood. Here we describe the features of this organism, together with the complete genome sequence and annotation. This is the first completed genome sequence of a member of the genus for which a complete genome sequence is described. The 2,749,646 bp long genome with its 2558 protein-coding and 71 RNA genes is part of the Genomic Encyclopedia of Bacteria and Archaea project.

\section{Introduction}

Strain Prevot 55134 (= DSM $20603=$ ATCC $14870=$ CIP 55.134) is the type strain of the species Jonesia denitrificans, the type species of the genus Jonesia [1]. The isolate originated from cooked ox blood [2]. J. denitrificans was originally placed into the genus Listeria and described as $L$. denitrificans by Prevot in 1961 [3], even though it differed morphologically from other members of the genus Listeria. Later, extensive analysis based on the determination of the $\mathrm{G}+\mathrm{C}$ content [4], DNADNA hybridization [4], peptidoglycan type [5,6], as well as fatty acids and polar lipid pattern $[5,7]$ confirmed the misclassification of the strain. As a consequence the strain was transferred to the new genus Jonesia by Rocourt et al. 1987 [1]. Five years later Stackebrandt and Prauser assigned Jonesia to the family Cellulomonadaceae despite being aware that Jonesia differed from members of other genera of the family e.g. in its $\mathrm{G}+\mathrm{C}$ content, peptidoglycan type and its composition of isoprenoid quinones. The rationale to place Jonesia into this family was based on the finding that the $16 \mathrm{~S}$ rRNA 
Pukall, et al.

gene sequence analysis showed close relationship to Promicromonospora and Cellulomonas at that time [8]. Following extensive phylogenetic in 1995, studies Jonesia was subsequently excluded from the family Cellulomonadacea and placed in the family Jonesiaceae, within the suborder Micrococcineae [9]. With Jonesia quinghaiensis, an environmental isolate from mud of a soda lake in China, a second species of the genus was described by Schumann et al. in 2004 [10]. Two additional environmental strains closely related to J. denitrificans, with 98\% and 99\% 16S rRNA gene sequence similarity have been reported. These organisms were isolated from the microbial community of feed batch reactors for composting of household biowaste [11]. Here we present a summary classification and a set of features for J. denitrificans Prevot 55134 ${ }^{\mathrm{T}}$ together with the description of the complete genomic sequencing and annotation.

\section{Classification and features}

Figure 1 shows the phylogenetic neighborhood of $J$. denitrificans strain Prevot 55134 $4^{\mathrm{T}}$ in a 16S rRNA based tree. The sequences of the five 16S rRNA gene copies in the genome of strain Prevot 55134 do not differ from each other, and differ by eight nucleotides from the previously published $16 \mathrm{~S}$ rRNA gene sequence of DSM 20603 (X78420).

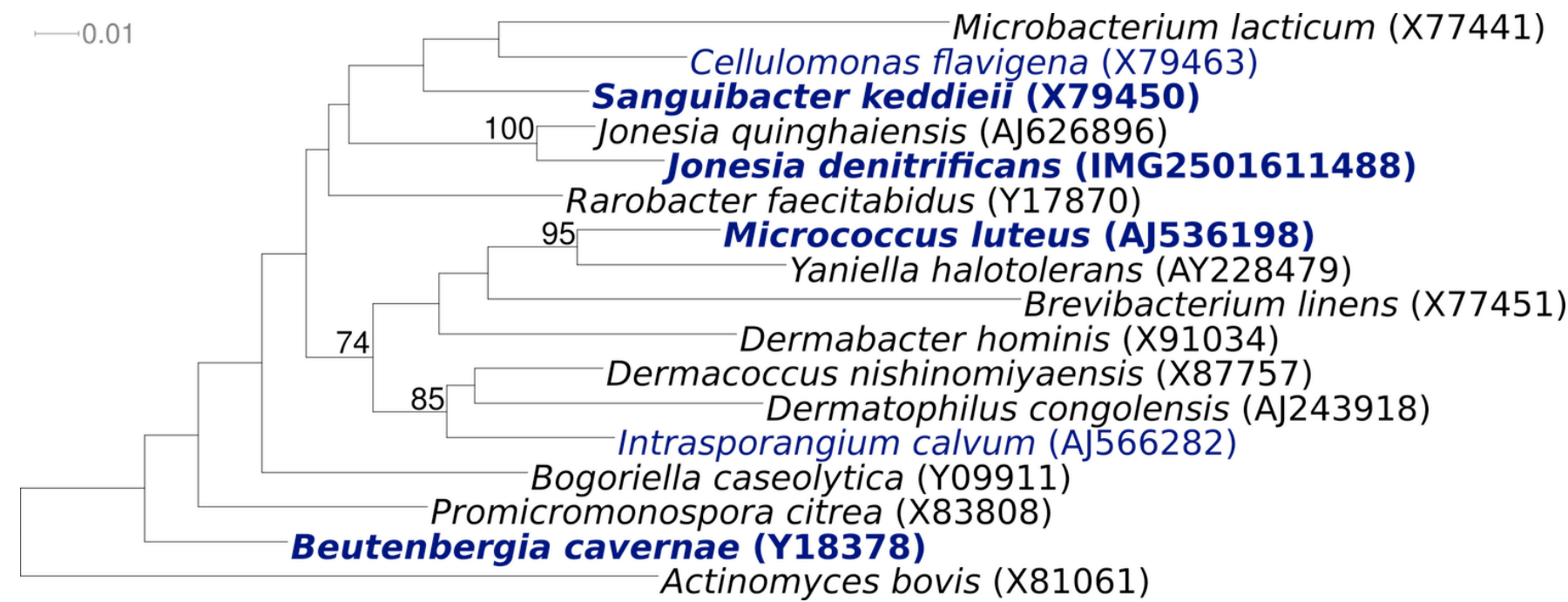

Figure 1. Phylogenetic tree highlighting the position of $J$. denitrificans Prevot $55134^{\top}$ relative to all type strains of the genus Jonesia and the type strains of all families within suborder Micrococcineae, inferred from 1,417 aligned characters $[19,20]$ of the 16S rRNA sequence under the maximum likelihood criterion [21]. Rooting was done with the type strain of Actinomyces bovis, the type species of Actinomyces, which is the type genus of the order Actinomycetales. The branches are scaled in terms of the expected number of substitutions per site. Numbers above branches are support values from 1,000 bootstrap replicates if larger than 60\%. Lineages with type strain genome sequencing projects registered in GOLD [22] are shown in blue, published genomes in bold, e.g. the GEBA genomes Beutenbergia cavernae [23], and Sanguibacter keddieii [24].

J. denitrificans type strain cells are Gram-positive, typically coryneform and characterized by irregular rods, $0.3-0.5 \mu \mathrm{m}$ in diameter and $2-3 \mu \mathrm{m}$ in length. Coccoid forms occur in older cultures (Table 1),. No endospores are formed. (Figure 2). The cells are motile by means of peritrichous flagella (absent in Figure 2). Colonies range from 0.5 to 1.5 $\mathrm{mm}$ in diameter on BHI agar. The optimum temperature for growth is $30^{\circ} \mathrm{C}$ [16]. The organism is catalase positive, oxidase negative and utilizes Dcellobiose, D-galactose, D-sorbitol, turanose and acetic acid [10]. Cellulose, starch, DNA and RNA are hydrolyzed. In addition, J. denitrificans produces acid from a large variety of sugars, polysaccharides and other compounds as described by Seeliger and Jones in 1986 [16] and is capable of denitrification [25]. The natural habitat of the organism is not known, however, J. denitrificans is a known pathogen of rats and mice when injected intraperitoneally [16].

\section{Chemotaxonomy}

The cell wall of strain Prevot $55134^{\mathrm{T}}$ contains murein of type $A 4 \alpha$, composed of L-Lys-L-Ser-D- 
Glu only [5,6], type A11.48 according to the DSMZ catalogue of strains (http://www.dsmz.de/ microorganisms/main.php?content id=35). In addition to the amino sugars muramic acid and glucosamine, galactosamine was detectable in the hydrolysate of the cell walls of $J$. denitrificans [5]. 12Methyl-tetradonic acid (ai-C15:0) and hexadonic acid (C16:0) constituted the major cellular fatty acid, and minor amounts of 14-methyl-hexadonic acid (ai-C17:0) and tetradecanoic acid (C14:0) were also detected [6,7]. Diphosphatidylglycerol (DPG) and phosphatidylinositol (PI) were identified by TLC as the polar lipids [6] and menaquinone of the MK-9 type was detected as the major component.

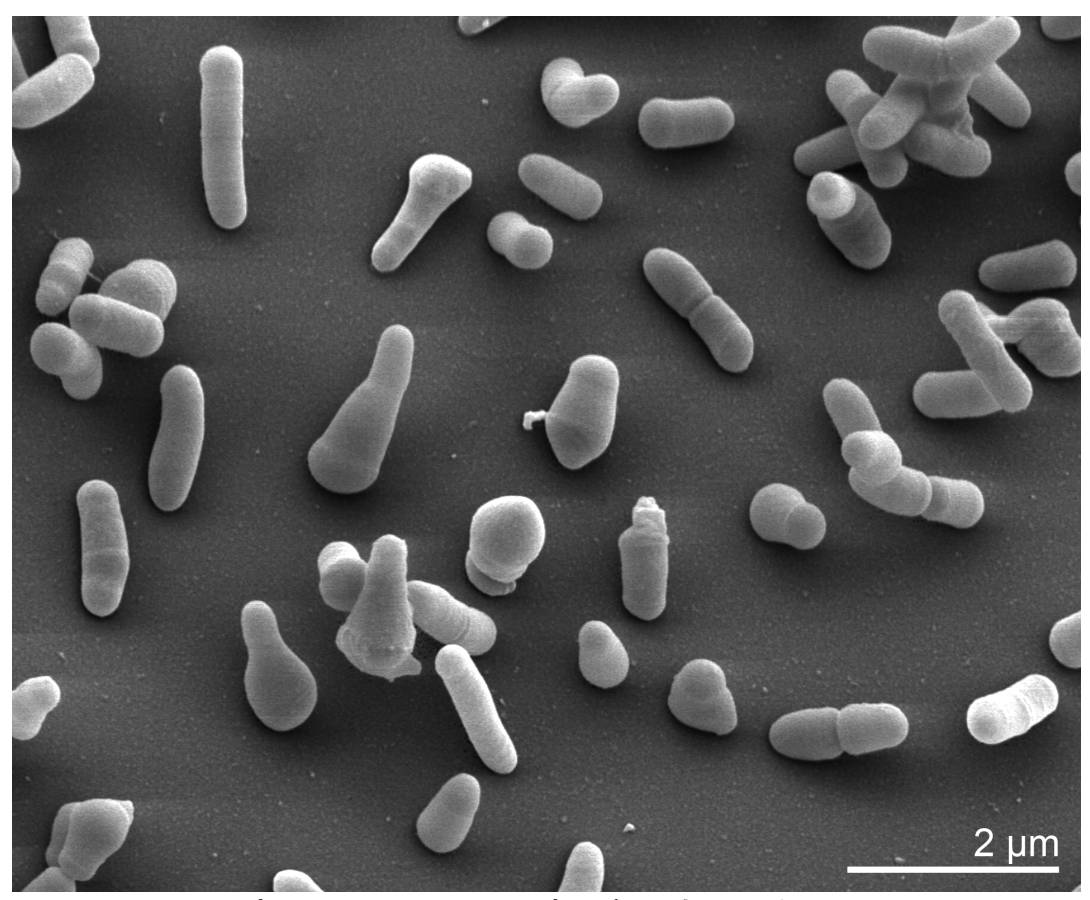

Figure 2. Scanning electron micrograph of J. denitrificans Prevot $55134^{\top}$ (Manfred Rohde, Helmholtz Centre for Infection Research (HZI), Braunschweig)

Table 1. Classification and general features of J. denitrificans Prevot $55134^{\top}$ in accordance with the MIGS recommendations [12]

\begin{tabular}{|c|c|c|c|}
\hline MIGS ID & Property & Term & Evidence code \\
\hline & & Domain Bacteria & TAS [13] \\
\hline & & Phylum Firmicutes & TAS [14] \\
\hline & & Class Actinobacteria & TAS [15] \\
\hline & & Subclass Actinobacteridae & TAS [15] \\
\hline & & Order Actinomycetales & TAS [15] \\
\hline & & Suborder Micrococcineae & TAS [15] \\
\hline & & Family Jonesiaceae & TAS [15] \\
\hline & & Genus Jonesia & TAS [1] \\
\hline & & Species Jonesia denitrificans & TAS [1] \\
\hline & & Type strain Prevot 55134 & TAS [16] \\
\hline & Gram stain & positive & TAS [16] \\
\hline & Cell shape & irregular rods, coccoid & TAS [16] \\
\hline & Motility & motile & TAS [16] \\
\hline & Sporulation & nonsporulating & TAS [16] \\
\hline & Temperature range & mesophile & TAS [16] \\
\hline & Optimum temperature & $30-37^{\circ} \mathrm{C}$ & TAS [16] \\
\hline & Salinity & $5 \% \mathrm{NaCl}$ & TAS [16] \\
\hline
\end{tabular}


Table 1. Classification and general features of $J$. denitrificans Prevot $55134^{\top}$ in accordance with the MIGS recommendations (cont.) [12]

\begin{tabular}{llll}
\hline MIGS ID & Property & Term & Evidence code \\
\hline MIGS-22 & Oxygen requirement & facultatively anaerobic & TAS [16] \\
& Carbon source & unknown & \\
& Energy source & unknown & \\
MIGS-6 & Habitat & natural habitat not known & TAS [16] \\
MIGS-15 & Biotic relationship & free living & NAS \\
MIGS-14 & Pathogenicity & pathogenic for animals & TAS [16] \\
& Biosafety level & 2 & TAS [17] \\
& Isolation & cooked ox blood & TAS [2] \\
MIGS-4 & Geographic location & France & \\
MIGS-5 & Sample collection time & not reported & \\
MIGS-4.1 & Latitude - Longitude & not reported & \\
MIGS-4.2 & Depth & not reported & \\
MIGS-4.3 & Altitude & not reported & \\
MIGS-4.4 & & & \\
\hline
\end{tabular}

Evidence codes - IDA: Inferred from Direct Assay (first time in publication); TAS: Traceable Author Statement (i.e., a direct report exists in the literature); NAS: Non-traceable Author Statement (i.e., not directly observed for the living, isolated sample, but based on a generally accepted property for the species, or anecdotal evidence). These evidence codes are from the Gene Ontology project [18]. If the evidence code is IDA, then the property was directly observed for a living isolate by one of the authors or an expert mentioned in the acknowledgements.

\section{Genome sequencing information} Genome project history

This organism was selected for sequencing on the basis of its phylogenetic position, and is part of the Genomic Encyclopedia of Bacteria and Archaea project. The genome project is deposited in the Genomes OnLine Database [22] and the complete genome sequence in GenBank Sequencing, finishing and annotation were performed by the DOE Joint Genome Institute (JGI). A summary of the project information is shown in Table 2.

Table 2. Genome sequencing project information

\begin{tabular}{lll}
\hline MIGS ID & Property & Term \\
\hline MIGS-31 & Finishing quality & Finished \\
MIGS-28 & Libraries used & Two Sanger libraries $-8 \mathrm{~kb}$ \\
MIGS-29 & SMCL200 and fosmid pcc1 Fos \\
MIGS-31.2 & Sequencing platforms & ABI3730 \\
MIGS-30 & Assemblers coverage & $9.5 x$ Sanger \\
MIGS-32 & Gene calling method & phrap \\
& INSDC ID & Prodigal, GenePRIMP \\
& Genbank Date of Release & August 27, 2009 \\
& GOLD ID & Gc01092 \\
& NCBI project ID & 20833 \\
& Database: IMG-GEBA & 2501533218 \\
MIGS-13 & Source material identifier & DSM 20603 \\
& Project relevance & Tree of Life, GEBA \\
\hline
\end{tabular}




\section{Growth conditions and DNA isolation}

J. denitrificans strain Prevot 55134T, DSM 20603, was grown in DSMZ medium 215 (BHI broth) [26], at $37^{\circ} \mathrm{C}$. DNA was isolated from $0.5-1 \mathrm{~g}$ of cell paste using the JGI CTAB-Protocol with a modified protocol for cell lysis (ALM), according to $\mathrm{Wu}$ et al. [27].

\section{Genome sequencing and assembly}

The genome was sequenced using only the Sanger platform. All general aspects of library construction and sequencing performed at the JGI can be

\section{Genome annotation}

Genes were identified using Prodigal [29] as part of the Oak Ridge National Laboratory genome annotation pipeline, followed by a round of manual curation using the JGI GenePRIMP pipeline (http://geneprimp.jgi-psf.org) [30]. The predicted CDSs were translated and used to search the National Center for Biotechnology Information (NCBI) non-redundant database, UniProt, TIGRFam, Pfam, PRIAM, KEGG, COG, and InterPro databases. Additional gene prediction analysis and functional annotation was performed within the found at the JGI website (http://www. jgi.doe.gov/). All reads were assembled using the phrap assembler. Possible mis-assemblies were corrected with Dupfinisher or transposon bombing of bridging clones [28]. Gaps between contigs were closed by editing in Consed, custom primer walk or PCR amplification. A total of 653 Sanger finishing reads were produced. The error rate of the completed genome sequence is less than 1 in 100,000 . Together all sequenced reads provided $9.5 \mathrm{x}$ coverage of the genome. The final assembly consists of 35,028 Sanger 454 reads.

Integrated Microbial Genomes Expert Review (IMG-ER) platform [31].

\section{Genome properties}

The single replicon genome is $2,749,646$ bp long with a $58.4 \%$ GC content (Table 3 and Figure 3 ). Of the 2,629 genes predicted, 2,558 were protein coding genes, and 71 RNAs. In addition, 47 pseudogenes were also identified. The majority of the genes $(68.3 \%)$ were assigned with a putative function, while the remaining ones are annotated as hypothetical proteins. The distribution of genes into COGs functional categories is presented in Table 4.

Table 3. Genome Statistics

\begin{tabular}{lrr}
\hline Attribute & \multicolumn{1}{c}{ Value } & \% of Total \\
\hline Genome size (bp) & $2,749,646$ & $100.00 \%$ \\
DNA coding region (bp) & $2,530,061$ & $92.01 \%$ \\
DNA G+C content (bp) & $1,606,252$ & $58.42 \%$ \\
Number of replicons & 1 & \\
Extrachromosomal elements & 0 & \\
Total genes & 2,629 & $100.00 \%$ \\
RNA genes & 71 & $2.92 \%$ \\
rRNA operons & 5 & \\
Protein-coding genes & 2,558 & $97.08 \%$ \\
Pseudo genes & 47 & $1.78 \%$ \\
Genes with function prediction & 1,796 & $68.31 \%$ \\
Genes in paralog clusters & 223 & $8.46 \%$ \\
Genes assigned to COGs & 1,830 & $69.45 \%$ \\
Genes assigned Pfam domains & 1,849 & $70.17 \%$ \\
Genes with signal peptides & 615 & $23.34 \%$ \\
Genes with transmembrane helices & 682 & $25.88 \%$ \\
CRISPR repeats & 0 & \\
\hline
\end{tabular}




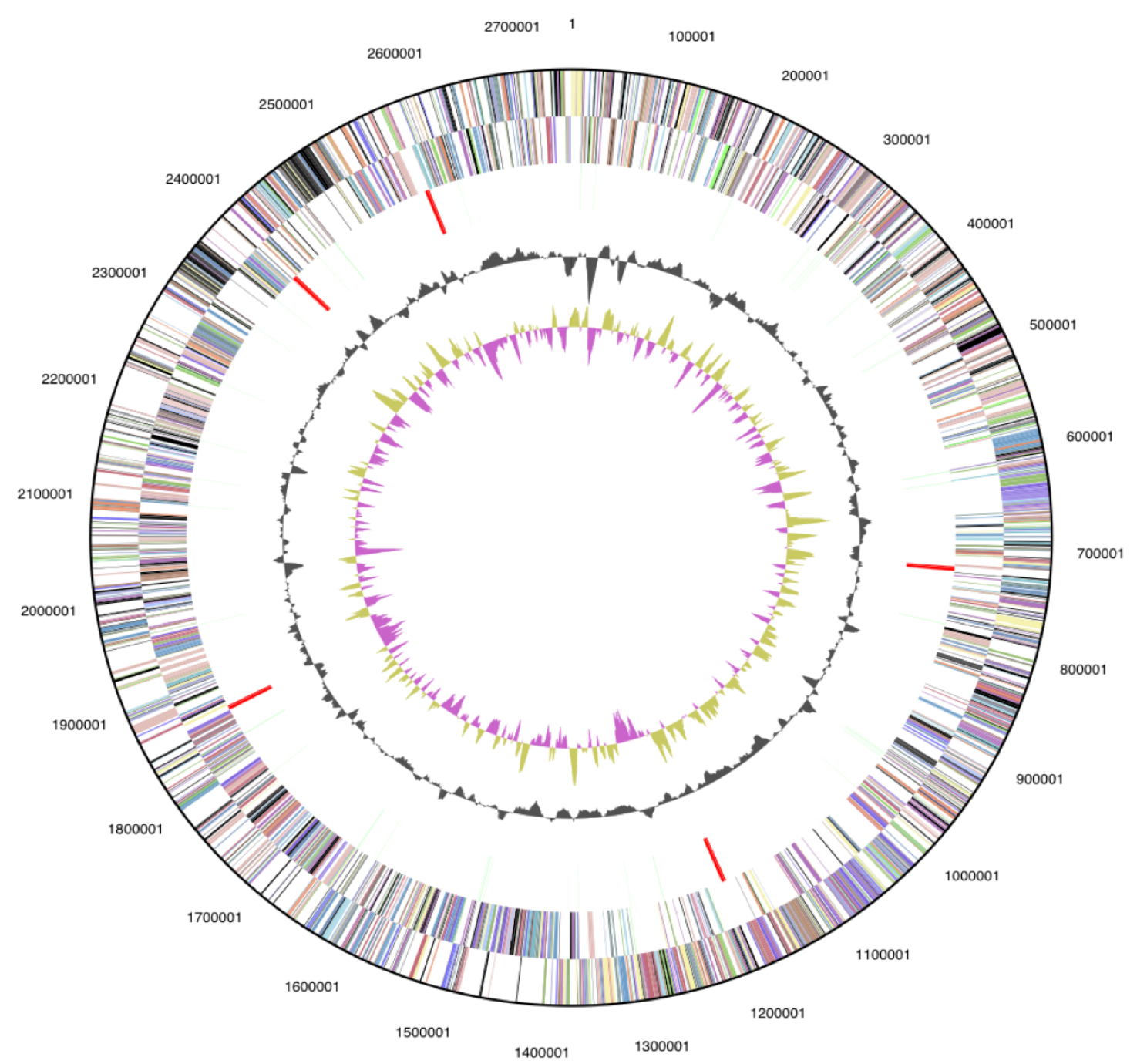

Figure 3. Graphical circular map of the genome. From outside to the center: Genes on forward strand (color by COG categories), Genes on reverse strand (color by COG categories), RNA genes (tRNAs green, rRNAs red, other RNAs black), GC content, GC skew.

Table 4. Number of genes associated with the general COG functional categories

\begin{tabular}{llll}
\hline Code & value & \%age & Description \\
\hline J & 144 & 5.6 & Translation, ribosomal structure and biogenesis \\
A & 1 & 0.0 & RNA processing and modification \\
K & 160 & 6.3 & Transcription \\
L & 100 & 3.9 & Replication, recombination and repair \\
B & 1 & 0.0 & Chromatin structure and dynamics \\
D & 22 & 0.9 & Cell cycle control, mitosis and meiosis \\
Y & 0 & 0.0 & Nuclear structure \\
V & 53 & 2.1 & Defense mechanisms \\
T & 89 & 3.5 & Signal transduction mechanisms \\
M & 93 & 3.6 & Cell wall/membrane biogenesis \\
N & 46 & 0.0 & Cell motility \\
Z & 0 & 0.0 & Cytoskeleton \\
W & 0 & 0.0 & Extracellular structures \\
U & 42 & 1.6 & Intracellular trafficking and secretion \\
\hline
\end{tabular}


Table 4. Number of genes associated with the general COG functional categories

\begin{tabular}{llll}
\hline Code & value & \%age & Description \\
\hline O & 79 & 3.1 & Posttranslational modification, protein turnover, chaperones \\
C & 110 & 4.3 & Energy production and conversion \\
G & 210 & 8.2 & Carbohydrate transport and metabolism \\
E & 162 & 6.3 & Amino acid transport and metabolism \\
F & 68 & 2.7 & Nucleotide transport and metabolism \\
H & 93 & 3.6 & Coenzyme transport and metabolism \\
I & 54 & 1.1 & Lipid transport and metabolism \\
P & 121 & 4.7 & Inorganic ion transport and metabolism \\
$\mathrm{Q}$ & 23 & 0.9 & Secondary metabolites biosynthesis, transport and catabolism \\
$\mathrm{R}$ & 207 & 8.1 & General function prediction only \\
$\mathrm{S}$ & 141 & 5.5 & Function unknown \\
- & 728 & 28.5 & Not in COGs \\
\hline
\end{tabular}

\section{Acknowledgements}

We gratefully acknowledge the help of Susanne Schneider (DSMZ) for DNA extraction and quality analysis. This work was performed under the auspices of the US Department of Energy's Office of Science, Biological and Environmental Research Program, and by the University of California, Lawrence Berkeley National

\section{References}

1. Rocourt J, Wehmeyer U, Stackebrandt E. Transfer of Listeria denitrificans to a new genus, Jonesia gen. nov., as Jonesia denitrificans comb. nov. Int J Syst Bacteriol 1987; 37:266-270.

2. Prévot $\mathrm{S}$. Traité de bactériologie systématique, Dunod, Paris, 1961

3. Sohier R, Benazet F, Piechaud M. Sur un germe du genre Listeria apparemment non pathoène. Ann Inst Pasteur (Paris) 1948; 74:54-57.

4. Stuart SE, Welshimer HJ. Intrageneric relatedness of Listeria Pirie. Int J Syst Bacteriol 1973; 23:8-14.

5. Fiedler F, Seger J. The murein types of Listeria grayi, Listeria murrayi and Listeria denitrificans. Syst Appl Microbiol 1983; 4:444-450.

6. Collins MD, Feresu S, Jones D. Cell wall, DNA base composition and lipid studies on Listeria denitrificans (Prévot). FEMS Microbiol Lett 1983;

18:131-134. doi:10.1111/j.15746968.1983.tb00464.x

7. Bernard KA, Bellefeuille M, Ewan EP. Cellular fatty acid composition as an adjunct to the identification of asporogenous, aerobic gram-positive rods. J Clin Microbiol 1991; 29:83-89. PubMed
Laboratory under contract No. DE-AC02-05CH11231, Lawrence Livermore National Laboratory under Contract No. DE-AC52-07NA27344, and Los Alamos National Laboratory under contract No. DE-AC0206NA25396, as well as German Research Foundation (DFG) INST 599/1-1.

8. Stackebrandt E, Prauser H. The family Cellulomonadaceae. In: The Prokaryotes, $2^{\text {nd }}$ edition, Springer Verlag 1992, Vol. 2:1323-1345.

9. Rainey FA, Weiss N, Stackebrandt E. Phylogenetic analysis of the genera Cellulomonas, Promicromonospora, and Jonesia and proposal to exclude the genus Jonesia from the family Cellulomonadaceae. Int J Syst Bacteriol 1995; 45:649-652. PubMed

10. Schumann P, Cui X, Stackebrandt E, Kroppenstedt $\mathrm{RM}, \mathrm{Xu} \mathrm{L}$, Jiang C. Jonesia quinghaiensis sp. nov., a new member of the suborder Micrococcineae. Int J Syst Evol Microbiol 2004; 54:2181-2184. PubMed doi:10.1099/ijs.0.63223-0

11. Hiraishi A, Narihiro T, Yamanaka Y. Microbial community dynamics during start-up operation of flowerpot-using fed-batch reactors for composting of household biowaste. Environ Microbiol 2003; 5:765-776. PubMed doi:10.1046/j.14622920.2003.00473.x

12. Field D, Garrity G, Gray T, Morrison N, Selengut J, Sterk P, Tatusova T, Thomson N, Allen MJ, Angiuoli SV, et al. Towards a richer description of our complete collection of genomes and metagenomes: the "Minimum Information about a Genome Sequence" (MIGS) specification. Nat Bio- 
Pukall, et al.

technol 2008; 26:541-547. PubMed

doi:10.1038/nbt1360

13. Woese CR, Kandler O, Wheelis ML. Towards a natural system of organisms: proposal for the domains Archaea, Bacteria, and Eucarya. Proc Natl Acad Sci USA 1990; 87: 4576-4579. PubMed doi:10.1073/pnas.87.12.4576

14. Garrity GM, Holt JG. Taxonomic Outline of the Archaea and Bacteria. Bergey's Manual of Systematic Bacteriology 2nd ed. (D.R. Boone and R.W. Castenholz, eds.), Springer-Verlag, New York 2001; 1:155-166.

15. Stackebrandt E, Rainey FA, Ward-Rainey NL. Proposal for a new hierachic classification system, Actinobacteria classis nov. Int I Syst Bacteriol 1997; 47:479-491.

16. Seeliger HPR, Jones D. The genus Listeria. In Bergey's Manual of Systematic Bacteriology, Williams and Wilkins 1986, Vol. 2:1235-1245.

17. Anonymous. Biological Agents: Technical rules for biological agents www.baua.de TRBA 466.

18. Ashburner M, Ball CA, Blake JA, Botstein D, Butler $\mathrm{H}$, Cherry JM, Davis AP, Dolinski K, Dwight SS, Eppig JT, et al. Gene ontology: tool for the unification of biology. The Gene Ontology Consortium. Nat Genet 2000; 25:25-29. PubMed doi:10.1038/75556

19. Lee C, Grasso C, Sharlow MF. Multiple sequence alignment using partial order graphs. Bioinformatics 2002; 18:452-464. PubMed doi:10.1093/bioinformatics/18.3.452

20. Castresana J. Selection of conserved blocks from multiple alignments for their use in phylogenetic analysis. Mol Biol Evol 2000; 17:540-552. PubMed

21. Stamatakis A, Hoover P, Rougemont J. A rapid bootstrap algorithm for the RAxML web-servers. Syst Biol 2008; 57:758-771. PubMed doi:10.1080/10635150802429642

22. Liolios K, Mavromatis K, Tavernarakis N, Kyrpides NC. The Genomes OnLine Database (GOLD) in 2007: status of genomic and metagenomic projects and their associated metadata. Nucleic
Acids Res 2008; 36:D475-D479. PubMed

doi:10.1093/nar/gkm884

23. Land M, Pukall R, Abt B, Göker M, Rohde M, Glavina Del Rio T, Tice H, Copeland A, Cheng JF, Lucas $\mathrm{S}$, et al. Complete genome of Beutenbergia cavernae type strain $\left(\mathrm{HKI} 0122^{\mathrm{T}}\right)$. Stand Genomic Sci 2009; 1:21-28. doi:10.4056/sigs. 1162

24. Ivanova N, Sikorski J, Sims D, Brettin T, Detter JC, Han C, Lapidus A, Copeland A, Glavina Del Rio T, Nolan M, et al. Complete genome of Sanguibacter keddieii type strain (ST-74 $\left.{ }^{\top}\right)$. Stand Genomic Sci 2009; 1:110-118.. doi:10.4056/sigs.16197

25. Zumft WG. The denitrifying Prokaryotes. In: The Prokaryotes, Springer Verlag 1992, Vol. 1:554582.

26. List of growth media used at DSMZ: http://www.dsmz.de/microorganisms/ media_list.php

27. Wu M, Hugenholtz P, Mavromatis K, Pukall R, Dalin E, Ivanova N, Kunin V, Goodwin L, Wu M, Tindall BJ, et al. A phylogeny-driven genomic encyclopedia of Bacteria and Archaea. (In press).

28. Sims D, Brettin T, Detter JC, Han C, Lapidus A, Copeland A, Glavina Del Rio T, Nolan M, Chen F, Lucas S, et al. Complete genome of Kytococcus sedentarius type strain $\left(541^{\mathrm{T}}\right)$. Stand Genomic Sci 2009; 1:12-20. doi:10.4056/sigs.761

29. Anonymous. Prodigal Prokaryotic Dynamic Programming Genefinding Algorithm. Oak Ridge National Laboratory and University of Tennessee 2009. http://compbio.ornl.gov/prodigal/

30. Pati A, Ivanova N, Mikhailova N, Ovchinikova G, Hooper SD, Lykidis A, Kyrpides NC. GenePRIMP: A Gene Prediction Improvement Pipeline for microbial genomes. (Submitted) 2009

31. Markowitz VM, Mavromatis K, Ivanova NN, Chen IMA, Chu K, Kyrpides NC. Expert IMG ER: A system for microbial genome annotation expert review and curation. Bioinformatics 2009; 25:22712278. PubMed doi:10.1093/bioinformatics/btp393 